\title{
Planejamento da designação de maquinistas a trens de viagem de longa distância em ferrovias de transporte de carga
}

\author{
Planning the assignment of train drivers to long distance travel trains on \\ freight transport railways
}

\author{
Franco Collodetti Mazioli ${ }^{1}$, Rodrigo de Alvarenga Rosa ${ }^{2}$, Matheus da Silva Gravel ${ }^{3}$, \\ Vivian Parreira 4 \\ 'Universidade Federal do Espírito Santo, Espírito Santo - Brasil, francomazioli@gmail.com \\ 2Universidade Federal do Espírito Santo, Espírito Santo - Brasil, a.rosa@ufes.br \\ ${ }^{3}$ Vale S.A. - Brasil, matheus.gravel@vale.com \\ ${ }^{4}$ Vale S.A. - Brasil, vivian.parreira@vale.com
}

\section{Recebido:}

28 de janeiro de 2020

Aceito para publicação:

4 de fevereiro de 2020

Publicado:

16 de novembro de 2020

Editor de área:

Marcio D'Agosto

\section{Palavras-chaves:}

Designação de maquinistas.

Operação ferroviária.

Transporte ferroviário.

\section{Keywords:}

Crew management.

Railway optimization.

Railway operation.

Railway transport.

DOI:10.14295/transportes.v28i4.2027

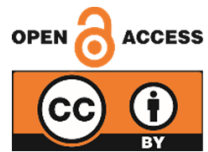

\begin{abstract}
RESUMO
As ferrovias brasileiras transportam cargas em viagens longas. O trem é conduzido por um maquinista e, por conta da extensão da viagem com tempo total de duração, geralmente, superior à escala de trabalho do maquinista, fazendo-se necessário realizar a troca de maquinistas durante a viagem em locais pré-definidos, denominados destacamentos. Na literatura, este problema é tratado de forma separada por dois subproblemas: Crew Scheduling e Crew Rostering. Diferentemente, este artigo propõe um modelo matemático para resolver de forma integrada os dois subproblemas, inspirado no Problema de Roteamento de Veículos com Múltiplas Viagens e Múltiplos Depósitos, para planejar a designação de maquinistas para conduzir os trens que passarão pelos destacamentos, buscando minimizar o pagamento de salários e horas extras aos maquinistas. Os resultados demonstram possíveis reduções de custos quando comparados com o planejamento manual da ferrovia analisada e reduções de custo podem ser alcançadas se aumentar o tempo de escala.
\end{abstract}

\begin{abstract}
Brazilian railroads carry loads through very long trips. A train is driven by a driver and, due to the long trips, the total trip time of the trains is generally higher than the period of the driver's shift and, thus it is necessary to change drivers during the trip in predefined places, called detachments. In the literature, this problem is solved separately by two subproblems: Crew Scheduling and Crew Rostering. Differently, this paper proposes a mathematical model to solve the two subproblems in an integrated way, inspired by the Vehicle Routing Problem of with Multiple Trips and Multiple Depots, to plan the designation of train drivers to drive the trains that will pass through the detachments aiming to minimize the cost with the payment of train drivers. Results show possible cost reductions when compared to the manual planning of the analyzed railway and increasing the shift period, cost reductions can be achieved.
\end{abstract}

\section{INTRODUCÃO}

De acordo com o Ministério dos Transportes, as ferrovias brasileiras são responsáveis por mais de 20\% das cargas transportadas no país (CNT, 2017). No Brasil, no ano de 2017, as ferrovias movimentaram cerca de 538 milhões de toneladas úteis, representando um crescimento de 
1,07\% em relação ao ano de 2016, segundo dados da Agência Nacional de Transportes Terrestres (ANTT, 2018).

Um dos maiores custos da operação ferroviária é o pagamento de salários e horas extras aos maquinistas que são profissionais especializados em conduzir os trens. Para cada trem que irá circular na ferrovia, um maquinista deve ser designado para conduzi-lo. As ferrovias brasileiras que transportam cargas possuem grandes extensões e o tempo de viagens dos trens desde sua origem até o seu destino é, via de regra, superior ao tempo de uma escala de trabalho do maquinista. Assim, as ferrovias são divididas em trechos intercalados por destacamentos, onde os maquinistas são lotados. Desta forma, faz-se necessário, na maioria das vezes, realizar a troca destes profissionais durante a viagem do trem. Cada maquinista realiza uma viagem por escala, conduzindo um trem entre dois destacamentos contíguos, sendo um deles sempre a sua sede. Por questões de segurança a troca de maquinista ocorre sempre em um destacamento. Também devem ser designados maquinistas para os trens que iniciam a viagem no trecho do destacamento.

No problema estudado nesse artigo, os maquinistas trabalham em regime de escala, sendo cada uma com seis horas de duração. Durante um dia, diversos trens são programados para circular na ferrovia em horários distintos. A cada destacamento que o trem passa, deve se proceder a troca de maquinista. Assim, em um dia, com base nos trens programados e com os maquinistas disponíveis nas várias escalas e destacamentos, deve se proceder a designação dos maquinistas aos trens de forma a minimizar o pagamento de salários e horas extras aos maquinistas.

Esse artigo propõe um modelo matemático inspirado no Problema de Roteamento de Veículos com Múltiplas Viagens e Múltiplos Depósitos (PRV-MD-MV) (BRAEKERS, RAMAEKERS e NIEUWENHUYSE 2016; LIN et al. 2014) adaptado para resolver o problema de designação de maquinistas a trens que realizam viagens de longa distância. 0 modelo tem por objetivo a minimização dos custos devidos ao pagamento de salários e horas extras aos maquinistas. Na literatura, este problema é solucionado de forma separada por meio de dois subproblemas: 1) Crew Scheduling e 2) Crew Rostering. Diferentemente, o modelo matemático proposto nesse artigo soluciona o problema de forma integrada.

Para avaliar o modelo proposto o mesmo foi aplicado a Estrada de Ferro Vitória a Minas (EFVM). A EFVM está situada nos Estados do Espírito Santo e Minas Gerais possui uma extensão de 895 km de via (ANTT, 2018). No ano de 2018, a EFVM movimentou 124 milhões de TKU. Na EFVM, a designação de maquinistas a trens é realizada de forma manual e, portanto, propostas de ferramentas de otimização que venham a apoiar o planejamento da designação de maquinistas são importantes para reduzir o tempo de planejamento, bem como apresentar soluções que possam levar a redução dos custos com maquinistas, i.e., redução do número de maquinistas e redução de horas extras.

A Seção 2 apresenta o Problema de Gerenciamento de Tripulação e uma revisão bibliográfica. Na Seção 3, é apresentado o modelo matemático proposto. Na Seção 4, apresentam-se as instâncias de testes. Na Seção 5 são apresentados os resultados e análises dos mesmos. Por fim, na Seção 6, tem-se as conclusões.

\section{PROBLEMA DE GERENCIAMENTO DE TRIPULAÇÃO}

O Problema de Gerenciamento de Tripulação (Crew Management Problem) tem por finalidade a 
elaboração das escalas de trabalho das equipes necessárias para atender o cronograma de planejamento de viagens e, usualmente, objetiva a redução do número de empregados necessários para realização do planejamento (CAPRARA et al., 1997). O Crew Management Problem é decomposto em dois subproblemas: 1) Crew Scheduling e 2) Crew Rostering. 0 subproblema Crew Scheduling elabora uma lista de tarefas, geralmente viagens, que serão atribuídas a cada tripulação. 0 subproblema de Crew Rostering trata da alocação das tarefas geradas no Crew Scheduling a cada tripulação (ERNST et al., 2001). A seguir é apresentada uma revisão bibliográfica sobre os principais artigos publicados sobre o Crew Management Problem que trataram do transporte ferroviário.

Caprara et al. (1997) apresentaram uma solução por Relaxação Lagrangeana e os resultados alcançados foram melhores que os obtidos pelo planejamento manual da ferrovia estudada. Caprara, Fischetti e Toth (1999) apresentaram um sistema para o planejamento de tripulação, onde o problema é dividido em 3 etapas, aplicando métodos heurísticos para alcançar uma solução.

Ernst et al. (2001) propuseram um modelo de duas etapas para otimização dos problemas Crew Scheduling e Crew Rostering. 0 modelo permite que as equipes se desloquem como passageiros, além disso, possibilita que sejam percorridos até três trechos de distância em relação à sede das equipes, assim como a sequência de atividades atendidas seja concluída num destacamento diferente do qual estão lotados. 0 modelo foi testado com dados de uma ferrovia australiana de transporte de cargas. Os resultados mostraram uma redução do número de empregados necessários para o planejamento em relação às práticas da empresa.

Jütte, Albers e Thonemann (2011) desenvolveram uma solução baseada em Geração de Colunas para atender a demanda de uma empresa alemã de transporte ferroviário de carga. A designação de atividades é feita diariamente e todo maquinista inicia e conclui suas atividades no mesmo destacamento. Jütte e Thonemann (2012) apresentaram também uma solução baseada em Geração de Colunas para tratar o Crew Scheduling Problem. O método proposto decompõe o problema em regiões sobrepostas que são otimizadas em paralelo.

Hanafi e Kozan (2014) propuseram uma meta-heurística Simulaed Annealing para tratar o Crew Scheduling Problem, onde definiam a sequência de atividades para atender a programação de trens de carga. No problema tratado o maquinista só pode terminar sua escala no seu destacamento sede. $\mathrm{O}$ objetivo era minimizar os números de tarefas, reduzindo o tempo ocioso das equipes na transição entre elas. Jütte e Thonemann (2015) acrescentaram ao problema descrito por Jütte e Thonemann (2012) a possibilidade dos maquinistas se deslocarem entre estações sem necessariamente estarem operando um trem. Eles apresentaram um algoritmo de decomposição baseado em Graph Partitioning.

As produções científicas encontradas sobre o tema propõem modelos e algoritmos destinados a atender a casos muito específicos e frequentemente não podem ser aplicados para diferentes situações. 0 presente artigo apresenta um modelo mais genérico que pode facilmente ser adaptado a qualquer ferrovia do mundo.

Os artigos publicados não podem garantir a otimização do Crew Management Problem, pois eles abordam o problema, dividindo-o nos dois subproblemas citados anteriormente, Crew Scheduling e Crew Rostering, e então resolver cada um dos dois subproblemas. Neste artigo, diferentemente dos demais artigos publicados, o Crew Management Problem é tratado em uma única etapa, sem considerar o agrupamento de tarefas para serem realizadas em uma escala, 
que é o Crew Scheduling, para posterior alocação destas às tripulações. Ou seja, é estudado somente a etapa de Crew Rostering designando maquinistas a várias tarefas, i.e., viagens de trens, sem agrupá-las previamente, podendo então garantir a otimização do Crew Management Problem como um todo. Também, nesse artigo, é adotada uma abordagem inovadora inspirada no PRV-MD-MV para desenvolver o modelo matemático proposto.

\section{DESCRIÇÃO DO PROBLEMA ESTUDADO}

No transporte ferroviário de minério de ferro no Brasil, em geral, os trens realizam viagens de longa distância, acima de $800 \mathrm{~km}$, com tempo de duração longo, acima de 12 horas de viagem. Desta forma, os tempos de viagem dos trens são superiores ao tempo de uma escala do maquinista, 6 horas, fazendo-se necessário revezar os maquinistas para poder concluir a viagem do trem que é analisada em uma sequência de viagens por trechos menores. Estes trechos são definidos de tal forma que se possa aproveitar ao máximo o tempo de escala dos maquinistas. Assim, entre cada um desses trechos, um destacamento é definido. Cada trem, originalmente realiza uma viagem de longa distância e, para tratamento pelo modelo matemático, ele é dividido em vários trens que viajam de um destacamento para outro.

A EFVM, ferrovia analisada nesse artigo, conta com 6 destacamentos ao longo da ferrovia (Figura 1). Cada destacamento conta com um quadro de maquinistas para atender a demanda diária de condução dos trens que passam ou se originam nele.

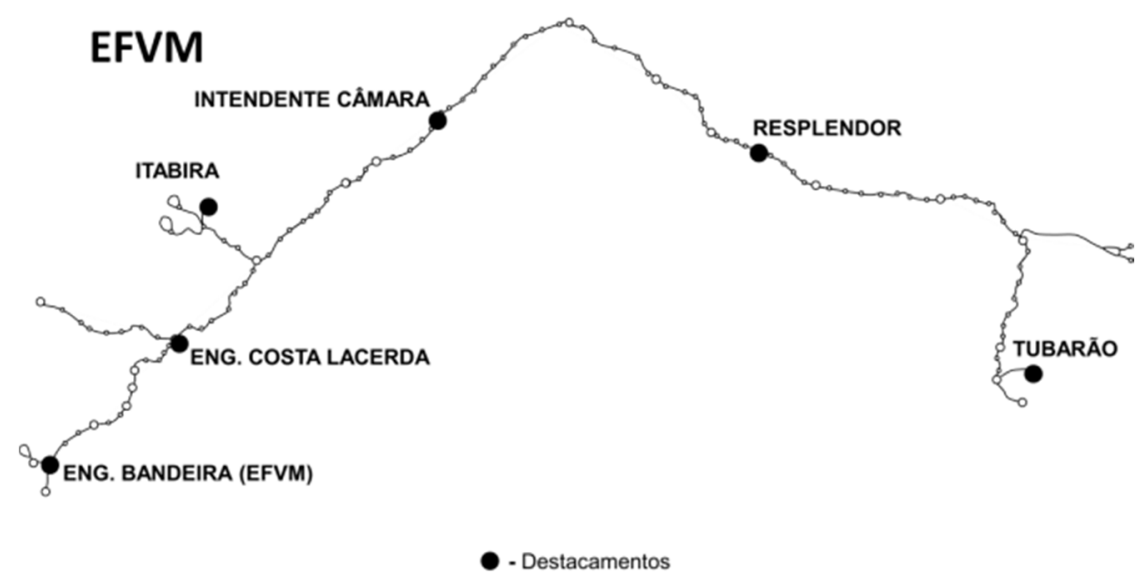

Figura 1. Destacamentos EFVM

Os maquinistas realizam viagens do destacamento onde estão lotados, denominados de destacamento sede, para outro destacamento contiguo a esse como mostrado na Figura 2. Ele conduz somente um trem em cada escala. Com base nas informações da ferrovia estudada, foram adotadas como premissas que os maquinistas trabalham em no máximo três trechos, Figura 2. Uma situação específica que ocorre na EFVM, e que pode ocorrer em outras ferrovias de carga, é que entre o destacamento de Costa Lacerda e a mina de Brucutu, o maquinista conduz um trem saindo do destacamento de Costa Lacerda viajando até a mina onde percorre a pera ferroviária, esperando o trem ser carregado com minério de ferro, e retorna para o destacamento de origem do trem sem haver a troca do maquinista durante o trajeto. Esse tipo de viagem é denominado de vai e volta. 


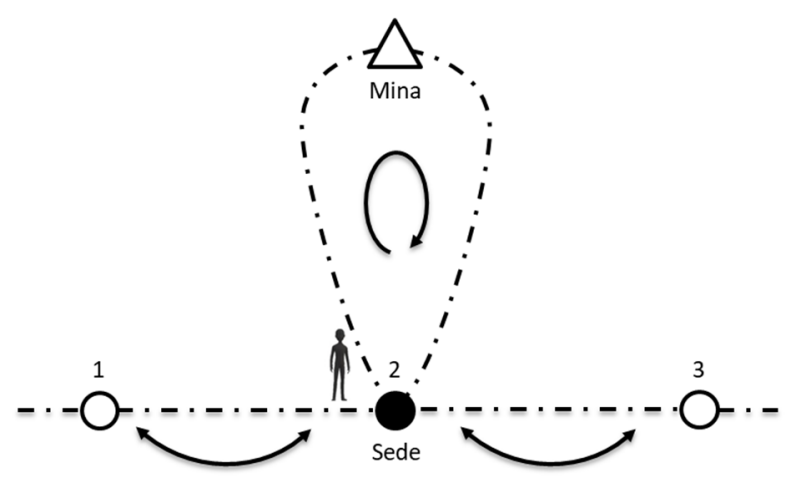

Figura 2. Possíveis trechos atendidos pelos maquinistas

Os maquinistas após realizarem uma viagem devem dispor de um período de tempo para descansar entre escalas. Este período pode ser de dois tipos: 1) Descanso e 2) Folga. À medida que as escalas são cumpridas o maquinista recebe o Descanso entre escalas. Ao atingir o limite máximo de escalas consecutivas que ele pode realizar, o maquinista deve entrar na Folga. Os tempos de Descanso e de Folga adotados pela EFVM são 10 horas e 48 horas, respectivamente, sendo que ambos têm um limite máximo de 2 horas além do tempo adotado para o retorno ao trabalho. 0 limite máximo de escalas consecutivas é igual a 4. Os maquinistas trabalham escalas de 6 horas e possuem um limite máximo de horas trabalhadas dentro do trem de 10 horas. Na EFVM, o planejamento da designação dos maquinistas a trens é realizado por uma equipe especializada da ferrovia, de forma manual, baseando-se na experiência desses profissionais.

\section{MODELO MATEMÁTICO PROPOSTO}

O modelo matemático proposto para planejar a designação de maquinistas aos trens tomou como base o Problema de Roteamento de Veículos, com Múltiplos Depósitos e com Múltiplas Viagens (PRV-MD-MV). Para adaptar o PRV-MD-MV ao problema abordado, considerou-se que cada maquinista é um depósito. Neste depósito, existe um único veículo que inicia uma viagem para atender clientes, que são os diversos trens que demandam maquinistas. Ao fim desta viagem, este veículo segue para um depósito virtual, correspondente ao depósito real, onde a escala se encerra. Esta interpretação pode ser vista na Figura 3.

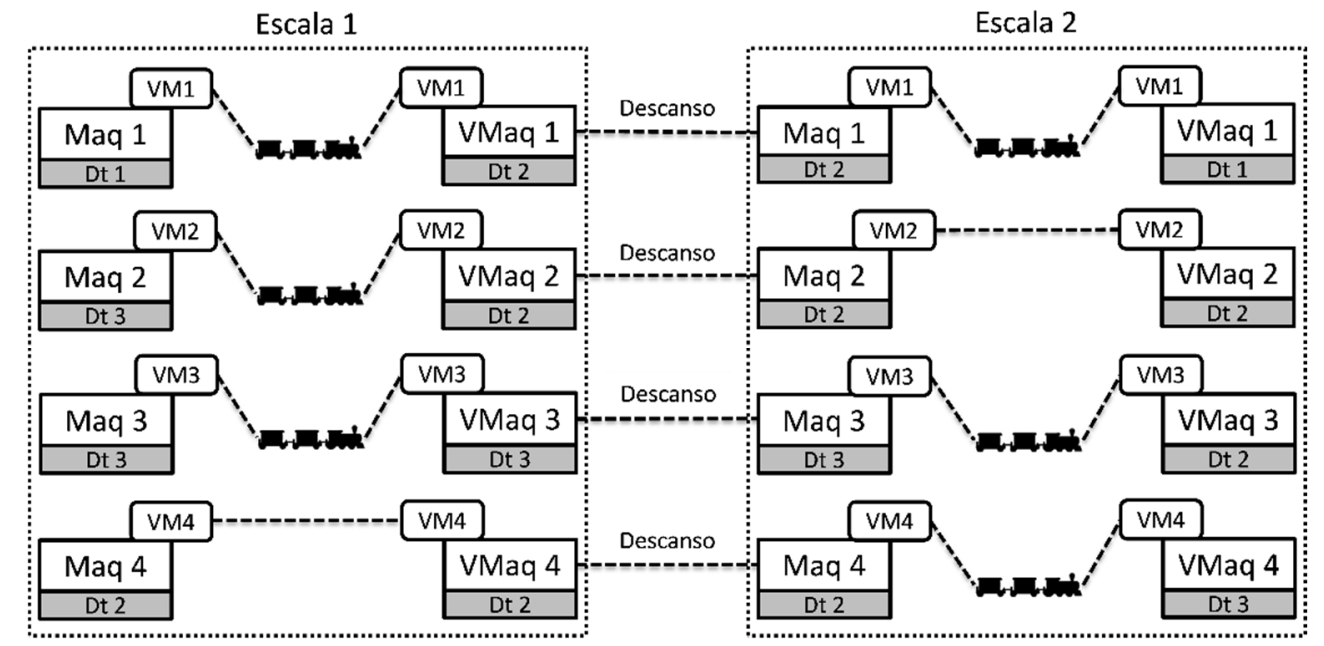

Legenda: Maq-Maquinista; VM-Veículo Maquinista; VMaq-Maquinista Virtual; Dt - Destacamento

Figura 3. Esquema do modelo matemático proposto 
No modelo matemático proposto, cada trem é caracterizado com seu destacamento de origem e o de destino em cada trecho. Para um maquinista ser alocado a um trem, ambos devem estar no mesmo local de início da viagem, assim como o trem deve estar destinado para viajar para um destacamento que o maquinista esteja habilitado a atender. À medida que os maquinistas conduzem os trens, o destino do trem passa a ser o destacamento onde o maquinista realizará o Descanso ou a Folga, como descrito anteriormente. Com isso, é possível identificar o novo local que ele se encontra para quando ele regressar para próxima escala possibilitando definir quais os trens que ele poderá conduzir. No modelo proposto, assim como na prática, podem existir maquinistas que não serão designados para atender nenhum trem na sua escala de trabalho. No entanto, nenhum trem pode deixar de ser atendido, ou seja, ter um maquinista designado para ele.

Uma viagem do veículo corresponde a designação do maquinista, representado pelo veículo, ao trem que ele conduzirá. Ela é limitada ao tempo máximo de permanência do maquinista no trem. Um veículo/maquinista pode ou não atender trens em uma viagem e, caso o maquinista não atenda nenhum trem, é considerado que o tempo de viagem do veículo é igual ao período de uma escala de trabalho do maquinista (Maq 4 na Escala 1, Figura 3). Ao fim da viagem, o veículo/maquinista chega ao seu respectivo depósito virtual e, no caso do problema de designação de maquinistas, o veículo deve ficar parado neste depósito até o início da próxima viagem, representando o descanso ou folga entre escalas, Figura 3.

0 modelo matemático proposto é apresentado em cinco partes, a saber: os conjuntos, os parâmetros, as variáveis de decisão, a função objetivo e as restrições. Considerando nm, o número de maquinistas disponíveis, $n t$, o número de viagens de trens a serem atendidas, ne, o número máximo de designações dos maquinistas, $n f$, o número de faixas de designação e $n p$, o número de possíveis trechos que o maquinista pode atender, os conjuntos do modelo matemático são:

$M R \quad$ Conjunto dos maquinistas reais, $M R=\{1, \ldots, n m\}$;

NT Conjunto dos trens, $N T=\{(n m+1), \ldots,(n m+n t)\}$;

$M V \quad$ Conjunto dos maquinistas virtuais, $M V=\{(1+n m+n t), \ldots,(2 n m+n t)\}$;

TMR Conjunto dos maquinistas reais e trens, $T M R=\{1, \ldots,(n m+n t)\}$;

TMV Conjunto dos maquinistas virtuais e trens, $T M V=\{(n m+1), \ldots,(2 n m+n t)\}$;

TMT Conjunto de todos os nós, $T M T=\{1, \ldots,(2 n m+n t)\}$;

$E \quad$ Conjunto de designações, $E=\{1, \ldots, n e\}$;

EA Conjunto auxiliar de designação, não considerando a primeira escala, $E A=\{2, \ldots, n e\}$

EB Conjunto auxiliar de designação, não considerando a última escala, $E B=\{1, \ldots,(n e-1)\}$

$D \quad$ Conjunto auxiliar para definir um trecho que o maquinista pode ser designado a trem, $D=\{1,2\}$. Sendo o primeiro o destacamento sede do maquinista e o segundo um possível destacamento que define o trecho que o maquinista pode ser designado a trem; $\left|E^{*}\right|$ é o módulo dinâmico da mistura,;

$O P \quad$ Conjunto de possíveis trechos a serem atendidos por um maquinista $O P=\{1, \ldots, n p\}$;

$F \quad$ Conjunto de faixas de designação $F=\{1, \ldots, n f\}$; 
Os parâmetros do modelo são:

$t v_{i} \quad$ Tempo gasto no nó $i \in T M T$ no caso dos nós pertencentes à $N T$, este parâmetro representa o tempo de viagem do trem. No caso dos nós pertencentes à $M R \mathrm{e}$ à $M V$, esse tempo é igual a 0 ;

$a_{i} \quad$ Momento de início da viagem do trem $i \in N T$;

$s^{k} \quad$ Momento de chegada do maquinista no destacamento, $k \in M R$;

$t l \quad$ Tempo máximo de trabalho de um maquinista por escala;

te $\quad$ Limite de tempo de uma escala do maquinista;

$t d \quad$ Tempo de descanso após o término de uma escala;

tf Tempo de folga após atender uma sequência de designações;

lr $\quad$ Limite de tempo para retornar da folga e/ou do descanso;

ot $_{i} \quad$ Destacamento de origem do trem $i \in N T$;

$d t_{i} \quad$ Destacamento de destino do trem $\mathrm{i} \in \mathrm{NT}$;

$d m^{k, d, p} \quad$ Trecho $p \in O P$ entre destacamentos $d \in D$ que o maquinista $k \in M R$ pode trabalhar. Sendo que o destacamento sede será sempre quando $d=1$. Ele estabelece uma matriz para os possíveis trechos que o maquinista pode trabalhar.

$l s^{k, f} \quad$ Limite superior da faixa, $f \in F$, de designação de um maquinista $k \in M R$

$l i^{k, f} \quad$ Limite inferior da faixa, $f \in F$, de designação de um maquinista $k \in M R$;

$s h^{k} \quad$ Valor da hora extra paga ao maquinista $k \in M R$;

$\mathrm{sm}^{k} \quad$ Salário do maquinista $k \in M R$;

$M \quad$ Valor grande para lógica do modelo;

$m \quad$ Valor pequeno para lógica do modelo.

Como mencionado, existem dois tipos de período para descansar entre escalas: 1) Descanso e 2) Folga. Para estabelecer o tipo de período é determinada sua faixa de designação, $f \in F$, que indica a quantidade de escalas que o maquinista irá cumprir antes desse período. Cada faixa de designação é caracterizada com um limite inferior $l i^{k, f}$ e um limite superior $l s^{k, f}$ do número de escalas. A EFVM estabeleceu que o limite superior da faixa de designação é de 4 escalas. 0 Descanso ocorre ao final de cada escala enquanto o maquinista não atinge o limite superior da faixa. A Folga ocorre ao término da última escala igual ao limite superior da faixa.

As variáveis de decisão do modelo matemático são:

$x_{i, j}^{k, e} \quad$ Variável binária que assume o valor igual a 1 se existe viagem do maquinista $k \in M R$ na designação $e \in E$, entre os nós $i, j \in T M T$ e, 0 caso contrário;

$\omega_{i}^{k, e} \quad$ Momento que o maquinista $k \in M R$, na escala $e \in E$, inicia a viagem, em um nó $i \in T M T$;

$l m^{k, e} \quad$ Destacamento onde o maquinista $k \in M R$ está na designação $e \in E$;

$u^{k} \quad$ Variável binária que assume o valor igual a 1 se o maquinista $k \in M R$ for designado para trabalhar em pelo menos uma escala e, 0 caso contrário; $h e^{k, e} \quad$ Horas extras do maquinista $k \in M R$, na escala $e \in E$.

$f t_{i}^{k, p, d}$

Variável binária que assume o valor 1 se o maquinista $k \in M R$ pode conduzir o trem $i \in N T$, viajando no trecho $p \in O P$, tendo início no destacamento $d \in D$ e, 0 caso contrário. 
Antes de definir as restrições, é necessário explicar a notação “ $\rightarrow$ "utilizada nas Restrições (21), (22), (25), (26) e (27) própria do ambiente IBM ILOG CPLEX Optimization Studio 12.8 (IBM, 2019), onde foi desenvolvido o modelo matemático e que representa uma restrição lógica com a função de implicação. Ou seja, utilizando esta notação, uma vez que a restrição lógica é atendida, lado esquerdo, ela implica que uma restrição do lado direito deve ser respeitada. Assim, este ambiente de desenvolvimento lineariza um modelo matemático não linear, permitindo que o CPLEX Versão 12.8 possa resolver o problema. A partir das descrições anteriores, são apresentadas a seguir a função objetivo e as restrições do modelo.

Função Objetivo:

Minimizar $\sum_{k \in M R} \sum_{e \in E} s h^{k} h e^{k, e}+\sum_{k \in M R} s m^{k} u^{k}$

Sujeito a:

$$
\begin{aligned}
& \sum_{j \in T M T} \sum_{e \in E} \sum_{k \in M R} x_{i, j}^{k, e}=1 \\
& \forall i \in N T \\
& \sum_{j \in T M V} \sum_{k a \in M R} x_{k, j}^{k a, e}=1 \\
& \sum_{j \in T M V} \sum_{\substack{k a \in M R \mid \\
k a \neq k}} x_{k, j}^{k a, e}=0 \\
& \sum_{i \in T M R} \sum_{k a \in M R} x_{i, k+n t+n m}^{k a, e}=1 \\
& \sum_{i \in T M R} \sum_{\substack{k a \in M R \mid \\
k a \neq k}} x_{i, k+n t+n m}^{k a, e}=0 \\
& \sum_{j \in T M T} \sum_{k a \in M R} \sum_{e \in E} x_{k+n t+n m, j}^{k a, e}=0 \quad \forall k \in M R \\
& \sum_{i \in T M T} \sum_{k a \in M R} \sum_{e \in E} x_{i, k}^{k a, e}=0 \\
& \sum_{j \in T M V} x_{i, j}^{k, e}-\sum_{j \in T M R} x_{j, i}^{k, e}=0 \\
& x_{i, i}^{k, e}=0 \\
& \sum_{i \in N T} \sum_{j \in T M V} x_{i, j}^{k, e} \geq 0 \\
& \sum_{i \in N T} \sum_{j \in T M V} x_{i, j}^{k, e} \leq 1 \\
& \omega_{j}^{k, e} \geq \omega_{i}^{k, e}+t v_{i} x_{i, j}^{k, e}-\left(1-x_{i, j}^{k, e}\right) M \\
& \forall k \in M R, e \in E \\
& \forall k \in M R, e \in E \\
& \forall k \in M R, e \in E \\
& \forall k \in M R, e \in E \\
& \forall k \in M R \\
& \forall i \in N T, k \in M R, e \in E \\
& \forall i \in T M T, k \in M R, e \in E \\
& \forall k \in M R, e \in E \\
& \forall k \in M R, e \in E \\
& \forall i \in T M R, j \in T M V, \quad k \in M R, \\
& e \in E
\end{aligned}
$$




$$
\begin{array}{ll}
\omega_{i}^{k, e}=a_{i}\left(x_{i, k+n t+n m}^{k, e}+\sum_{j \in N T} x_{i, j}^{k, e}\right) & \forall i \in N T, k \in M R, e \in E \\
\omega_{k+n t+n m}^{k, e}-\omega_{k}^{k, e} \leq t l & \forall k \in M R, e \in E \\
\omega_{k}^{k, 1}=s^{k} & \forall k \in M R \\
\omega_{k}^{k, e} \geq \omega_{k+n t+n m}^{k, e-1}+t f & \forall k \in M R, e \in E A, f \in F \mid \\
\omega_{k}^{k, e} \leq \omega_{k+n t+n m}^{k, e-1}+t f+l r & (e-1)=l s^{k, f} \\
& \forall k \in M R, e \in E A, f \in F \mid \\
\omega_{k}^{k, e} \geq \omega_{k+n t+n m}^{k, e-1}+t d & (e-1)=l s^{k, f} \\
& \forall k \in M R, e \in E A, f \in F \mid \\
\omega_{k}^{k, e} \leq \omega_{k+n t+n m}^{k, e-1}+t d+l r & \left(\left((e-1) \geq l i^{k, f}\right) \wedge\right. \\
\left(\sum_{i \in N T} \sum_{j \in T M V} x_{i, j}^{k, e}=0\right) \rightarrow \omega_{k+n t+n m}^{k, e}=\omega_{k}^{k, e}+t e & \left.\left.\left((e-1)<l s^{k, f}\right)\right)\right) \\
& \forall k \in M R, e \in E A, f \in F \mid \\
& \left(\left((e-1) \geq l i^{k, f}\right) \wedge\right. \\
& \left.\left.\left((e-1)<l s^{k, f}\right)\right)\right) \\
& \forall k \in M R, e \in E \\
&
\end{array}
$$$$
\left(\begin{array}{c}
\left(\operatorname{lm}^{k, e}=o t_{i}\right) \wedge \\
\left(\sum_{p \in O P} \sum_{d \in D} f t_{i}^{k, p, d} \geq 1\right) \wedge \\
\left(\sum_{r \in N T} \sum_{j \in T M V} x_{r, j}^{k, e}=1\right) \wedge \\
\left(\sum_{j \in M V} x_{i, j}^{k, e}=1\right)
\end{array}\right) \rightarrow
$$$$
\left(l m^{k, e+1}=d t_{i}\right)
$$$$
f t_{i}^{k, p, d}=1
$$$$
f t_{i}^{k, p, d}=0
$$$$
\left(\sum_{p \in O P} \sum_{d \in D} f t_{i}^{k, p, d}=0\right) \rightarrow\left(\sum_{j \in T M V} x_{i, j}^{k, e}=0\right)
$$$$
\left(\sum_{i \in N T} \sum_{j \in T M V} x_{i, j}^{k, e-1}=0\right) \rightarrow\left(l m^{k, e}=l m^{k, e-1}\right)
$$

$\forall k \in M R, e \in E$

$\forall k \in M R, i \in N T, e \in E B$

$\forall i \in N T, k \in M R, p \in O P$,

$d \in D \mid\left(d m^{k, d, p}=d t_{i}\right)$

$\forall i \in N T, k \in M R, p \in O P$,

$d \in D \mid\left(d m^{k, d, p} \neq d t_{i}\right)$

$\forall i \in N T, k \in M R, p \in O P, d \in$ $D$ 


$$
\begin{array}{ll}
\left(l m^{k, e} \neq o t_{i}\right) \rightarrow \sum_{j \in T M V} x_{i, j}^{k, e}=0 & \forall i \in N T, k \in M R, e \in E \\
l m^{k, 1}=d m^{k, 1,1} & \forall k \in M R \\
\sum_{j \in N T} \sum_{e \in E} x_{k, j}^{k, e} \geq m u^{k} & \forall k \in M R \\
\sum_{j \in N T} \sum_{e \in E} x_{k, j}^{k, e} \leq M u^{k} & \\
h e^{k, e} \geq \omega_{k+n t+n m}^{k, e}-\omega_{k}^{k, e}-\left(t e \sum_{j \in T M V} x_{k, j}^{k, e}\right) & \forall k \in M R \\
h e^{k, e} \leq t l-t e & \forall k \in M R, e \in E \\
u^{k} \in\{0,1\} & \forall k \in M R, e \in E \\
x_{i, j}^{k, e} \in\{0,1\} & \forall k \in M R \\
f t_{i}^{k, p, d} \in\{0,1\} & \forall i \in T M T, j \in T M T, k \in M R, \\
\omega_{i}^{k, e} \in \mathbb{R}^{+} & e \in E \\
h e^{k, e} \in \mathbb{R}^{+} & \forall i \in N T, k \in M R, p \in O P, d \in \\
& D \\
& \forall i \in T M T, k \in M R, e \in E \\
& \forall k \in M R, e \in E
\end{array}
$$

A função objetivo, Equação (1), representa a soma do pagamento das horas extras trabalhadas por todos os maquinistas e o pagamento de salário de todos os maquinistas que foram designados a pelo menos um trem. A hora extra é calculada como o tempo que o maquinista chega ao destacamento de destino menos o momento de início da escala do maquinista menos o tempo de escala determinado. A soma de ambos os pagamentos devem ser minimizadas.

As Restrições (2) garantem que cada trem $i \in N T$ é atendido apenas uma vez e por um único maquinista $k \in M R$. As Restrições (3) e (4) garantem que cada veículo virtual, equivalente ao maquinista $k \in M R$, na designação $e \in E$, sairá do seu respectivo depósito para atender um trem $i \in N T$ ou irá para seu depósito virtual correspondente. As Restrições (5), para cada veículo $k \in M R$ na designação $e \in E$, garante que um trem ou o depósito de origem do maquinista, $i \in T M R$, será o nó anterior ao depósito virtual de destino do veículo. As Restrições (6) determinam que apenas o veículo representando um determinado maquinista pode atender o depósito virtual do mesmo.

As Restrições (7) garantem que nenhuma viagem tem início nos depósitos virtuais e as Restrições (8) garantem que nenhuma viagem termine nos depósitos reais. As Restrições (9) garantem a conservação do fluxo. As Restrições (10) garantem que não ocorra um arco ligando o nó $i \in T M T$ para ele mesmo. As Restrições (11) e (12) definem se um maquinista é destinado a atender um trem numa designação ou não.

As Restrições (13) garantem que caso o nó $j \in T M V$ seja atendido após o nó $i \in T M R$ com o veículo $k \in M R$ na designação $e \in E$, então o instante de início do atendimento do nó $j \in T M V$ seja após o instante de início do atendimento do nó anterior $i \in T M R$ mais o tempo de viagem 
do mesmo. As Restrições (14) garantem que o maquinista $k \in M R$ começa a atender o trem $i \in$ NT no mesmo horário que o mesmo está disponível para iniciar a viagem. As Restrições (15) asseguram que o tempo de chegada ao depósito virtual menos o tempo de partida do veículo $k \in M R$, seja menor que o limite de tempo de trabalho de um maquinista por escala. As Restrições (16) assumem que o tempo de partida do veículo/maquinista $k \in M R$ na sua primeira designação deve ser maior ou igual ao momento de início da sequência de escalas que ele irá cumprir até a próxima folga.

As Restrições (17) e (18) definem se o maquinista irá cumprir folga, caso tenha realizado o máximo de designações para o período de planejamento. As Restrições (19) e (20) definem se o maquinista irá cumprir o descanso caso o maquinista tenha realizado menos designações que o limite estabelecido para o período de planejamento. Nas Restrições (21) caso o maquinista não tenha sido designado a atender um trem, então, o tempo de chegada ao depósito virtual é igual ao tempo de partida do veículo $k \in M R$ mais o tempo de uma escala te.

Caso as seguintes condições sejam todas verdadeiras: o destacamento atual do maquinista seja igual à origem do trem; o maquinista possa viajar para o destacamento de destino do trem; o maquinista conduza um trem nessa escala e o maquinista esteja designado para conduzir esse trem, então as Restrições (22) garantem que o destacamento que o maquinista estará na próxima designação será o mesmo que o destacamento do destino do trem.

As Restrições (23) e (24) definem se o destino do trem é igual a um dos possíveis destacamentos de trabalho do maquinista, $f t_{i}^{k, p, d}=1$, caso contrário, $f t_{i}^{k, p, d}=0$. As Restrições (25) garantem que caso o destino do trem $i \in N T$ seja diferente de um dos possíveis destacamentos que o maquinista $k \in M R$ pode atender, então o maquinista não poderá ser designado para este trem.

As Restrições (26) garantem que caso o maquinista não seja alocado a nenhum trem na escala, o destacamento da próxima escala será o mesmo da escala atual. As Restrições (27) garantem que caso o maquinista $k \in M R$ não esteja no mesmo destacamento de origem do trem $i \in$ $N T$, então o maquinista não poderá conduzir o trem. As Restrições (28) asseguram que a primeira designação do maquinista $k \in M R$ ocorra no seu destacamento sede. As Restrições (29) e (30) definem se o maquinista $k \in M R$ teve alguma designação a trem em ao menos uma das suas escalas, $u=1$ e zero caso contrário.

As Restrições (31) definem as horas extras realizadas por cada maquinista em cada uma de suas escalas. As Restrições (32) limitam a quantidade de horas extras por escala de cada maquinista, ao limite de tempo de trabalho em uma escala $(t l)$ menos o tempo de uma escala $(t e)$. As Restrições (33)-(37) definem o domínio das variáveis.

Com a definição do modelo matemático é possível calcular o número de variáveis de decisão inteiras e o de variáveis de decisão contínuas. Sendo as variáveis inteiras do modelo matemático: $x_{i, j}^{k, e}, l m^{k, e}, u^{k}$ e $f t_{i}^{k, p, d}$, então, o modelo possui $|T M T|^{2}|M R||E|+|M R||E|+|M R|+$ $|N T||M R\|O P\| D|$ variáveis de decisão inteiras. As variáveis contínuas são: $\omega_{i}^{k, e}$ e $h e^{k, e} \mathrm{e}$, portanto, o modelo possui $|T M T||M R||E|+|M R||E|$ variáveis de decisão contínuas. Sabendo que a quantidade de elementos do conjunto $|T M T|$ é igual a $2|M R|+|N T|$, então, é possível exprimir a quantidade de variáveis de decisão do modelo pelas Equações (38) e (39).

Número de variáveis de decisão inteiras:

$|M R|^{3}(4|E|)+|M R|^{2}(4|N T||E|)+|M R|\left(|N T|^{2}|E|+|N T||O P||D|+|E|+1\right)$ 
Número de variáveis de decisão contínuas:

$|M R|^{2}(2|E|)+|M R|(|N T||E|+|E|)$

\section{GERAÇÃO DE INSTÂNCIAS DE TESTES}

As instâncias para testar o modelo matemático proposto foram elaboradas com base nos dados levantados junto a EFVM. Foram desenvolvidas 13 instâncias, Tabela 1, variando: número de maquinistas, número de destacamentos, número de viagens de trens, número máximo de designações, tempo máximo de trabalho por escala e limite de tempo de uma escala do maquinista. Por questões de confidencialidade da EFVM, os valores referentes ao salário de cada maquinista, bem como os valores pagos de hora extra não podem ser divulgados.

As instâncias tiveram como objetivo realizar os testes para validação do modelo matemático proposto, assim como, analisar os resultados obtidos com base nas restrições estabelecidas, e a viabilidade da utilização do solver CPLEX 12.8 (IBM, 2018) para solucionar o problema. As instâncias foram apresentadas em grupos, sendo que cada grupo tem um objetivo específico explicado a seguir.

O Grupo 1 conta com a Instância 1 que foi criada para apresentar o comportamento do modelo, possuindo um tamanho reduzido para validação e apresentação visual dos resultados. 0 Grupo 2 possui duas instâncias, Instâncias 5 e 6, que foram desenvolvidas com dados reais da EFVM referentes ao destacamento de Costa Lacerda. 0 intuito é comparar os resultados do modelo com os resultados alcançados pelo planejamento manual realizado pela equipe da EFVM.

Os Grupos 3, 4 e 5, Instâncias de 7 a 15, foram criados com base em dados fornecidos pela EFVM. Elas possuem um tamanho próximo do real referente a um dia de operação considerando somente trens de minério de ferro. 0 Grupo 3 analisa o problema considerando o tempo máximo de trabalho por escala de 10 horas (padrão adotado pela EFVM) variando o tempo limite de escala em três valores nas três instâncias, respectivamente em: 6 horas (padrão adotado da EFVM), 7 horas e 8 horas. 0 Grupo 4 é similar ao Grupo 3, porém considera o tempo máximo de trabalho por escala de 11 horas. 0 Grupo 5 também é similar ao Grupo 3, porém considera o tempo máximo de trabalho por escala de 12 horas. Esses grupos têm a intenção de proporcionar a compreensão do impacto da variação do tempo de escala e o limite de tempo de trabalho de um maquinista em uma escala. As Instâncias 7 a 15 possuem cada uma 7.894 .935 variáveis inteiras e 37.800 variáveis contínuas. A quantidade de variáveis de decisão é igual entre as instâncias, uma vez que, a quantidade de maquinistas, viagens de trens e número de escalas não são alterados entre elas.

A Instância 17, Grupo 6, foi criada para testar a capacidade do CPLEX resolver instâncias de grande porte. Nesta instância foram considerados para o planejamento 60 maquinistas distribuídos em 6 destacamentos e 216 trens representando 2 dias de planejamento do grupo de maquinistas. A Instância 17 tem 27.173.100 variáveis inteiras e 80.880 variáveis contínuas.

No ambiente de desenvolvimento IBM ILOG CPLEX Optimization Studio 12.8, para o modelo matemático é gerado um arquivo com extensão *.mod e para cada instância é gerado um arquivo com extensão *.dat. No entanto, o solver CPLEX não consegue ler os arquivos *.mod e *.dat. Assim, o ambiente de desenvolvimento gera arquivos com extensão *.lp, com o modelo linearizado, que possuem a linguagem compreendida pelo solver CPLEX que resolverá a instância.

Na Tabela 2, pode-se notar que o tempo para geração do arquivo *.lp é muito elevado. Isso se 
deve, sobretudo, ao número de variáveis de decisão do modelo que, à medida que o número de maquinistas e de viagens de trens aumenta, ocorre um crescimento exponencial do número de variáveis de decisão do modelo. 0 número de variáveis inteiras e contínuas das 13 instâncias testadas são calculadas com base nas Equações 38 e 39 e podem ser vistos nas Tabela 2.

Tabela 1 - Dados das instâncias de teste e o número de variáveis de decisão

\begin{tabular}{|c|c|c|c|c|c|c|c|}
\hline \multirow{2}{*}{$\begin{array}{l}\text { 을 } \\
\text { 군 }\end{array}$} & \multirow{2}{*}{ 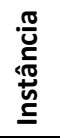 } & \multicolumn{6}{|c|}{ Parâmetros } \\
\hline & & $\begin{array}{l}\text { № de } \\
\text { Maq. }\end{array}$ & $\begin{array}{l}\text { № de } \\
\text { Trens }\end{array}$ & $\begin{array}{l}\text { № de } \\
\text { Destac. }\end{array}$ & $\begin{array}{l}\text { № de } \\
\text { Escalas }\end{array}$ & $\begin{array}{l}\text { Limite de tempo de } \\
\text { uma escala (h) }\end{array}$ & $\begin{array}{l}\text { Tempo máximo de trabalho de um } \\
\text { maquinista por escala }(\mathrm{h})\end{array}$ \\
\hline 1 & 1 & 6 & 13 & 3 & 4 & 6 & 10 \\
\hline \multirow{2}{*}{2} & 5 & 30 & 54 & 3 & 2 & 6 & 10 \\
\hline & 6 & 33 & 120 & 3 & 4 & 6 & 10 \\
\hline \multirow{3}{*}{3} & 7 & 45 & 119 & 6 & 4 & 6 & 10 \\
\hline & 8 & 45 & 119 & 6 & 4 & 7 & 10 \\
\hline & 9 & 45 & 119 & 6 & 4 & 8 & 10 \\
\hline \multirow{3}{*}{4} & 10 & 45 & 119 & 6 & 4 & 6 & 11 \\
\hline & 11 & 45 & 119 & 6 & 4 & 7 & 11 \\
\hline & 12 & 45 & 119 & 6 & 4 & 8 & 11 \\
\hline \multirow{3}{*}{5} & 13 & 45 & 119 & 6 & 4 & 6 & 12 \\
\hline & 14 & 45 & 119 & 6 & 4 & 7 & 12 \\
\hline & 15 & 45 & 119 & 6 & 4 & 8 & 12 \\
\hline 6 & 17 & 60 & 216 & 6 & 4 & 6 & 10 \\
\hline
\end{tabular}

Tabela 2 - Tamanho das instâncias de teste e o número de variáveis de decisão

\begin{tabular}{|c|c|c|c|c|c|}
\hline \multirow{2}{*}{ 을 } & \multirow{2}{*}{ 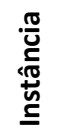 } & \multirow{2}{*}{ Tamanho arquivos *.Ip (Kb) } & \multicolumn{3}{|c|}{ Número de Variáveis de Decisão do Modelo } \\
\hline & & & Inteiras & Contínuas & Total \\
\hline 1 & 1 & $4.967,0$ & 15.498 & 624 & 16.122 \\
\hline \multirow{2}{*}{2} & 5 & $332.139,0$ & 789.570 & 6.900 & 796.470 \\
\hline & 6 & $5.366 .981,0$ & 4.590 .597 & 24.684 & 4.615 .281 \\
\hline \multirow{3}{*}{3} & 7 & $8.107 .902,0$ & 7.894 .935 & 37.800 & 7.932 .735 \\
\hline & 8 & $8.107 .902,0$ & 7.894 .935 & 37.800 & 7.932 .735 \\
\hline & 9 & $8.107 .902,0$ & 7.894 .935 & 37.800 & 7.932 .735 \\
\hline \multirow{3}{*}{4} & 10 & $8.107 .902,0$ & 7.894 .935 & 37.800 & 7.932 .735 \\
\hline & 11 & $8.107 .902,0$ & 7.894 .935 & 37.800 & 7.932 .735 \\
\hline & 12 & $8.107 .902,0$ & 7.894 .935 & 37.800 & 7.932 .735 \\
\hline \multirow{3}{*}{5} & 13 & $8.107 .902,0$ & 7.894 .935 & 37.800 & 7.932 .735 \\
\hline & 14 & $8.107 .902,0$ & 7.894935 & 37.800 & 7.932 .735 \\
\hline & 15 & $8.107 .902,0$ & 7.894 .935 & 37.800 & 7.932 .735 \\
\hline 6 & 17 & $52.475 .395,0$ & 27.173 .100 & 80.880 & 27.253 .980 \\
\hline
\end{tabular}

\section{RESULTADOS E ANÁLISES}

Para desenvolver o modelo e gerar o arquivo *.Ip foi utilizado o IBM ILOG CPLEX Optimization Studio 12.8. Para resolver o modelo matemático proposto, foi utilizado o solver IBM CPLEX Versão 12.8 (IBM, 2018). Ambos rodaram em um computador com dois processadores Intel ( 
Xeon® Silver 4116 CPU@ 2.10GHz+2.10GHz com 128 GB de memória RAM. Na Tabela 3 são apresentados o tempo de execução do CPLEX, o tempo de geração do arquivo *.Ip, o tempo total para resolver a instância, a função objetivo (FO), a quantidade de maquinistas utilizados e a quantidade de horas extras geradas.

Tabela 3 - Resultados alcançados pelo CPLEX

\begin{tabular}{|c|c|c|c|c|c|c|c|}
\hline Grupo & Inst. & $\begin{array}{l}\text { Tempo de Execução } \\
\text { CPLEX } \\
\text { (s) }\end{array}$ & $\begin{array}{l}\text { Tempo de } \\
\text { Geração do } \\
\text { Arquivo *.Ip } \\
\text { (s) }\end{array}$ & $\begin{array}{l}\text { Tempo Total } \\
\text { (s) }\end{array}$ & $\begin{array}{l}\text { FO } \\
\text { (R\$) }\end{array}$ & $\begin{array}{l}\text { Maqui. } \\
\text { Utilizados } \\
\text { (un) }\end{array}$ & $\begin{array}{c}\text { Horas } \\
\text { Extras } \\
\text { (h) }\end{array}$ \\
\hline 1 & 1 & 0,66 & 7,33 & 7,99 & $20.025,35$ & 5 & 8,3 \\
\hline \multirow{2}{*}{2} & 5 & 15,81 & 413,33 & 429,14 & $110.114,00$ & 30 & 0,0 \\
\hline & 6 & $1.019,25$ & $131.460,00$ & $132.479,25$ & $113.706,35$ & 30 & 0,0 \\
\hline \multirow{3}{*}{3} & 7 & $3.423,56$ & $38.486,10$ & $41.909,66$ & $116.537,85$ & 32 & 11,3 \\
\hline & 8 & $1.457,03$ & $22.554,10$ & $24.011,13$ & $115.430,80$ & 30 & 2,4 \\
\hline & 9 & $2.104,38$ & $30.403,10$ & $32.507,48$ & $113.275,00$ & 30 & 0,0 \\
\hline \multirow{3}{*}{4} & 10 & $5.220,27$ & $25.748,80$ & $30.969,07$ & $123.544,60$ & 32 & 10,8 \\
\hline & 11 & $1.842,97$ & $18.627,90$ & $20.470,87$ & $117.882,80$ & 30 & 2,4 \\
\hline & 12 & $2.673,38$ & $173.297,00$ & $175.970,38$ & $115.241,00$ & 30 & 0,0 \\
\hline \multirow{3}{*}{5} & 13 & $6.188,97$ & $199.202,00$ & $205.390,97$ & $123.485,45$ & 32 & 10,1 \\
\hline & 14 & $2.078,91$ & $18.455,60$ & $20.534,51$ & $115.818,90$ & 30 & 2,2 \\
\hline & 15 & $1.962,50$ & $19.978,00$ & $21.940,50$ & $115.934,00$ & 30 & 0,0 \\
\hline 6 & 17 & $22.719,89$ & $313.215,00$ & $335.934,89$ & $209.002,00$ & 54 & 0,0 \\
\hline
\end{tabular}

A Figura 4 ilustra a programação dos maquinistas relativo a Instância 1. Os maquinistas 1 e 2 (Maq 1 e Maq 2) são designados num primeiro momento para atender trens que irão fazer uma viagem do tipo vai e volta, ou seja, não mudam de destacamento ao final da escala. Em seguida os maquinistas cumprem o tempo de Descanso e retornam para escala seguinte. 0 maquinista 1 cumpre uma nova designação, enquanto o maquinista 2 não é designado para conduzir um trem, logo, permanece por 6 horas no destacamento, tempo de uma escala. Os maquinistas 1 e 2 podem realizar duas designações antes de entrar para a Folga de 48 horas. Após a Folga eles retornam para cumprir uma nova sequência de escalas na próxima faixa de designação.

Os maquinistas 3 e 4 são designados para atender trens em todas as 4 escalas antes de sair de Folga. 0 maquinista 5 não foi necessário para atender a demanda do planejamento, permanecendo no seu destacamento sede, cumprindo os tempos de cada escala e de descanso. Por fim, o maquinista 6 é designado para atender um trem na primeira escala, realizando uma viagem do tipo vai e volta. Após a designação ele cumpre o período de descanso e retorna na próxima escala. Como não há mais trens ele cumpre os tempos de escala e descanso até atingir o limite de escalas, entrando no período de folga.

No Grupo 2, têm-se as Instâncias 5 e 6 que representam situações reais do destacamento de Costa Lacerda e, portanto, é possível comparar os resultados alcançados pelo CPLEX com o resultado do planejamento manual realizado pela equipe da EFVM. No planejamento realizado pelo CPLEX, para as duas instâncias, foram necessários 30 maquinistas e nenhuma hora extra foi paga. Já a equipe de planejamento realizou a programação utilizando 32 maquinistas e pagando 8 horas extras na Instância 5 e de 33 maquinistas e pagando 9,5 horas extras na Instância 6. 


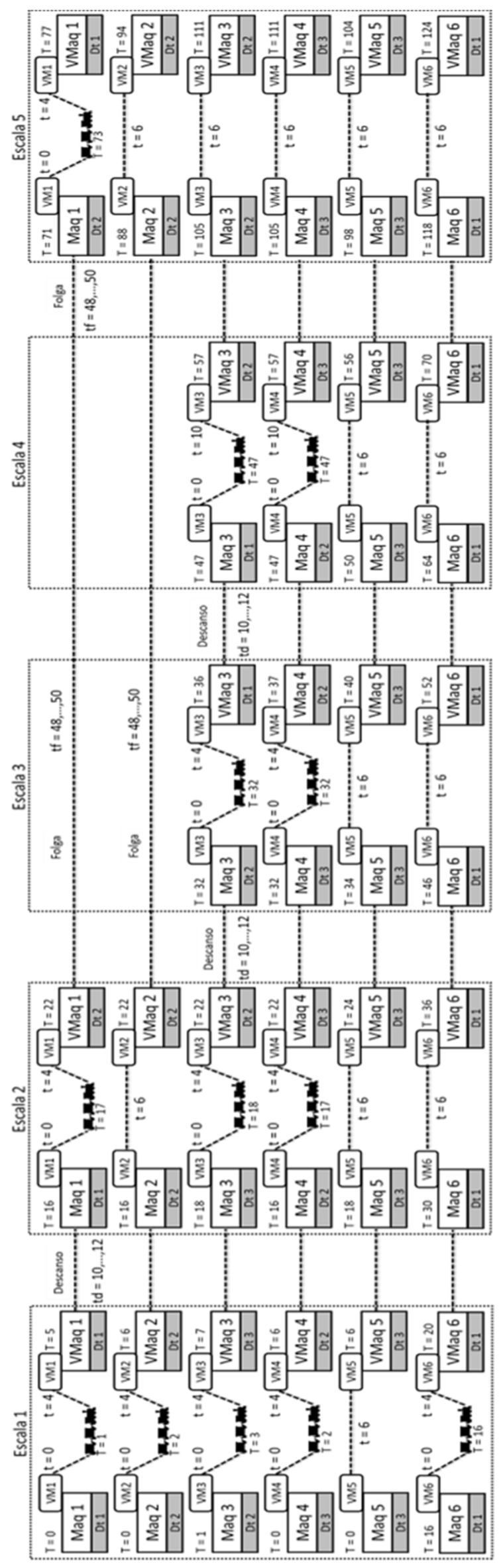

Figura 4. Esquema de solução da Instância 1 
Desta forma, pode-se perceber que caso o planejamento realizado pelo CPLEX para as duas instâncias do Grupo 2 fosse adotado pela EFVM, ela poderia diminuir o número de maquinistas utilizados em 2 e 3 respectivamente, assim como, reduzir o número de horas extras em 8 horas e 9,5 horas, respectivamente. Considerando que este planejamento representa um e dois dias de programação de um único destacamento, pode-se imaginar que em um ano esta redução poderia trazer economias significativas para a ferrovia.

As instâncias do Grupo 3 visam avaliar a variação do tempo de escala do maquinista, sendo que, respectivamente, as Instâncias 7, 8 e 9 possuem tempo de escala igual a 6 (escala utilizada pela ferrovia), 7 e 8 horas. As instâncias dos Grupos 4 e 5 seguem a mesmo padrão de variação do Grupo 3. 0 aumento do tempo de escala de 6 para 8 horas gerou uma redução de 2 maquinistas necessários para atender aos trens planejamento. Este padrão de redução se repetiu para os Grupos 4 e 5. Assim, apesar da dificuldade de se negociar com os sindicatos novos tempos de escala, este parâmetro deve ser avaliado com atenção pela ferrovia em uma futura negociação do acordo coletivo dos maquinistas, pois pode trazer ganhos importantes.

A análise entre os Grupos 3, 4 e 5 visa avaliar o impacto do tempo máximo de trabalho de um maquinista em uma escala e o limite de tempo de uma escala do maquinista. As Instâncias 7, 10 e 13 são praticamente iguais, variando o tempo máximo de trabalho de um maquinista em uma escala entre 10 (tempo utilizado pela ferrovia), 11 e 12, respectivamente. 0 mesmo ocorre com as Instâncias 8,11 e 12 e as Instâncias 9, 12 e 15.

Pode-se perceber que o número de maquinistas não se alterou nas soluções das Instâncias 7 , 10 e 13, i.e., 32 maquinistas. No entanto, a quantidade de horas extras teve uma ligeira diminuição de 11,3 horas, na Instância 7, para 10,1 horas na Instância 13. Este mesmo padrão de variação foi observado nas comparações das Instâncias 8, 11 e 12 e das Instâncias 9, 12 e 15. Isso mostra que para a ferrovia empreender esforços juntos aos sindicatos para alterar o tempo máximo de trabalho de um maquinista em uma escala não é em princípio algo que se justifique, pois os ganhos são pequenos em função do esforço de negociação com os sindicatos.

O Grupo 6 que contém a Instância 17, com 60 maquinistas, 216 trens, 6 destacamentos, foi criado para testar a capacidade do CPLEX em resolver instâncias de maior porte que se aproximam do planejamento da designação de maquinistas realizado para toda EFVM. O CPLEX alcançou a solução ótima com 54 maquinistas, sem pagar horas extras. No entanto, o tempo de processamento do IBM ILOG Optimization Studio para geração do arquivo *.Ip foi de 335.934,89 segundos (3,9 dias) e o tempo de execução do CPLEX foi de 22.719,89 segundos $(0,26$ dias ou 6,3 horas) perfazendo um total de 313.215,00 segundos entre geração e execução (4,2 dias), o que é um tempo não adequado para o planejamento do dia a dia da ferrovia.

Após as análises apresentadas anteriormente, o modelo matemático proposto mostrou ser capaz de resolver o problema de designação de maquinista e, quando comparado com os resultados obtidos pela ferrovia estudada, ele apresentou reduções no número de maquinistas e redução no número de horas extras geradas, o que levou a uma redução total dos pagamentos devidos aos maquinistas, o objetivo da gestão da ferrovia. No mais, o modelo conseguiu resolver instâncias de maior porte que representam o planejamento global de toda a ferrovia, porém necessitando um tempo computacional grande. A abordagem por meio do modelo PRV-MD-MV se mostrou robusta, sendo uma alternativa inovadora para resolver o problema Crew Management Problem. 


\section{CONCLUSÕES}

Este artigo analisou o problema de designação de maquinistas para conduzir trens, sendo esse problema complexo, muitas ferrovias carecem de ferramentas computacionais para realizar o planejamento dessa designação, apesar do custo com pagamento de salários e horas extras aos maquinistas ser um dos maiores custos da operação ferroviária. Na literatura, este problema é tratado de forma separada por dois subproblemas: Crew Scheduling e Crew Rostering. Diferentemente, este artigo propôs um modelo matemático para resolver de forma integrada os dois subproblemas, inspirado no Problema de Roteamento de Veículos com Múltiplas Viagens e Múltiplos Depósitos, para planejar a designação de maquinistas para atender aos trens que passarão pelos destacamentos buscando minimizar o custo com pagamento de salários e horas extras aos maquinistas.

O modelo matemático foi resolvido pelo CPLEX e os resultados sinalizam possíveis reduções de custos quando comparados com o planejamento manual da ferrovia analisada. Os resultados do CPLEX, no destacamento de Costa Lacerda, sugerem a possibilidade da redução de dois maquinistas, o que representa uma economia de custo importante, bem como redução do pagamento de horas extras.

Foram analisados dois parâmetros do problema: 1) Tempo de escala e 2) Tempo máximo de permanência no trem. Em relação ao tempo de escala, os resultados mostraram que um aumento neste parâmetro pode trazer reduções de custo importantes. Já o parâmetro Tempo máximo de permanência apresentou ganhos modestos. Para mudar qualquer um deles a ferrovia deve negociar com os sindicatos, o que é uma tarefa difícil. Assim, os resultados sugerem que é mais proveitoso uma negociação em aumento do tempo de escala de 6 para 8 horas.

Pode-se concluir que o modelo matemático proposto é uma ferramenta aplicável ao problema de planejamento da designação de maquinistas a trens da EFVM, podendo ser utilizado por qualquer ferrovia nacional ou internacional, sendo facilmente adaptável a qualquer outra restrição que porventura venha existir.

O modelo desempenhou bem seu objetivo de planejar a designação de maquinistas a trens, contudo seu tempo de processamento para instâncias próximas do real se tornou muito grande, em alguns casos dias, o que inviabiliza seu uso para planejamento diário da designação. Assim, sugere-se, como trabalho futuro, a elaboração de uma meta-heurística para resolver o modelo matemático proposto a fim de reduzir o tempo de processamento.

\section{AGRADECIMENTOS}

Os autores agradecem à FAPES (75528452/2016), ao CNPq (307797/2019-8) e CAPES pelo apoio financeiro.

\section{REFERÊNCIAS}

ANTT - Agência Nacional de Transportes Terrestres (2018). Disponível em: <http://www.antt.gov.br/>. (Acessado em: 06 de janeiro de 2018).

Braekers, K.; K. Ramaekers e I. V. Nieuwenhuyse (2016). The vehicle routing problem: State of the art classification and review. Computers \& Industrial Engineering, v. 99, p. 300-313. DOI: 10.1016/j.cie.2015.12.007

Caprara, A.; M. Fischetti; P. Toth; D. Vigo e P. L. Guida (1997). Algorithms for railway crew management. Mathematical Programming, v. 79, n. 1-3, p. 125-141. DOI: 10.1007/BF02614314

Caprara, A.; M. Fischetti e P. Toth (1999). A heuristic method for the set covering problem. Operations Research, v. 47, n. 5, p. 730-743. DOI: $10.1287 / o p r e .47 .5 .730$.

CNT. Confederação Nacional do Transporte. Anuário CNT do Transporte 2017 - Estatísticas Consolidadas. Disponível em: <http://anuariodotransporte.cnt.org.br/>. (Acesso em: 02 de fevereiro de 2018). 
Ernst, A. T.; H. Jiang; M. Krishnamoorthy; H. Nott e D. Sier (2001). An integrated optimization model for train crew management. Annals of Operations Research, v. 108, n. 1-4, p. 211-224. DOI: 10.1023/A:1016019314196

Hanafi, R. e E. Kozan (2014). A hybrid constructive heuristic and simulated annealing for railway crew scheduling. Computers \& Industrial Engineering, v. 70, p. 11-19. DOI: 10.1016/j.cie.2014.01.002

IBM. IBM ILOG CPLEX Optimization Studio 12.6. Using Logical Constraints. Disponível em: <https://www.ibm.com/support/knowledgecenter/pt-br/SSSA5P_12.7.1/ilog.odms.ide.help/OPL_Studio/opllanguser/topics/opl_languser_app_areas_cplex_using.html>. (Acesso em: 17 nov. 2017).

Jütte, S.; M. Albers; U. W. Thonemann e K. Haase (2011). Optimizing railway crew scheduling at DB Schenker. Interfaces, v. 41, n. 2, p. 109-122. DOI: $10.1287 /$ inte.1100.0549

Juette, S. e U. W. Thonemann (2012). Divide-and-price: A decomposition algorithm for solving large railway crew scheduling problems. European Journal of Operational Research, v. 219, n. 2, p. 214-223. DOI: 10.1016/j.ejor.2011.12.038

Jütte, S. e U. W. Thonemann (2015). A graph partitioning strategy for solving large-scale crew scheduling problems. OR Spectrum, v. 37, n. 1, p. 137-170. DOI: 10.1007/s00291-014-0381-8

Lin, C.; K. L. Choy; G. T. Ho; S. H. Chung e H. Y. Lam (2014). Survey of green vehicle routing problem: past and future trends. Expert Systems With Applications, v. 41, n. 4, p. 1118-1138. DOI: 10.1016/j.eswa.2013.07.107 